\title{
Associations among Bone Mineral Density, Physical Activity and Nutritional Intake in Middle-Aged Women with High Levels of Arterial Stiffness: A Pilot Study
}

\author{
Kanako Hamaguchi ${ }^{1}$, Toshiyuki Kurihara ${ }^{1}$, Masahiro Fujimoto ${ }^{2}$, Koji Sato ${ }^{3}$, \\ Motoyuki Iemitsu ${ }^{1}$, Takafumi Hamaoka ${ }^{4}$ (D) and Kiyoshi Sanada ${ }^{1, *(D)}$ \\ 1 College of Sport and Health Science, Ritsumeikan University, Shiga 525-8577, Japan; \\ gr0129xx@ed.ritsumei.ac.jp (K.H.); t-kuri@mti.biglobe.ne.jp (T.K.); iemitsu@fc.ritsumei.ac.jp (M.I.) \\ 2 National Institute of Advanced Industrial Science and Technology, Chiba 277-0882, Japan; \\ masahiro-fujimoto@aist.go.jp \\ 3 Graduate School of Human Development and Environment, Kobe University, Hyogo 657-8501, Japan; \\ sato712@people.kobe-u.ac.jp \\ 4 Department of Sports Medicine for Health Promotion, Tokyo Medical University, Nishi-Shinjuku 6-7-1, \\ Shinjuku-ku, Tokyo 160-0023, Japan; kyp02504@nifty.com \\ * Correspondence: ksanada@fc.ritsumei.ac.jp
}

Received: 1 February 2020; Accepted: 29 February 2020; Published: 3 March 2020

\begin{abstract}
There is little consensus regarding the impacts of physical activity and nutrient intake on bone mineral density (BMD) in subjects with high or low levels of arterial stiffness. This study was performed to investigate whether physical activity and nutrient intake are associated with BMD in middle-aged women with high levels of arterial stiffness. The study population consisted of middle-aged women aged 40-64 years $(n=22)$. BMD was assessed by dual-energy X-ray absorptiometry. Carotid-femoral pulse wave velocity (cf-PWV) was used as an indicator of arterial stiffness. Subjects were divided into two groups by median cf-PWV. Physical activity in free-living conditions was evaluated using a triaxial accelerometer. Nutrient intake was also measured using the brief-type self-administered diet history questionnaire. In the High-PWV group, BMD showed a significant negative correlation with age. Using a partial correlation model, BMD was associated with the number of steps and unsaturated fatty acid intake in the High-PWV group. These results suggest that BMD in middle-aged women with high levels of arterial stiffness may be associated with both the number of steps and nutritional intake. Recommendations of physical activity and nutritional intake for the prevention of osteopenia should include consideration of arterial stiffness.
\end{abstract}

Keywords: osteoporosis; nitric oxide; sedentary; hypertension; walking; oleic acid; linoleic acid; inflammation; antioxidant; postmenopausal women

\section{Introduction}

Osteoporosis, a chronic disease characterised by reduced bone mineral density (BMD), is one of the most prevalent factors contributing to fractures, mortality and mobility limitation in the elderly [1], and the prevalence in women is two- to sixfold higher than in men [2,3]. Treating established osteoporosis is difficult because drugs available for the prevention of osteoporosis may have long-term adverse effects and are often expensive. It is essential to prevent the development of this disease in the aging society. Age-related reductions in BMD are generally accelerated in women after menopause [2,3]. Age-related bone reduction occurs at a higher rate in middle age than in old age. Therefore, it 
is necessary to prevent bone loss in middle age. In general, age-related reductions in BMD are multifactorial. Therefore, optimisation of lifestyle-related factors known to influence BMD is an important strategy. Physical activity and adequate nutrition are key lifestyle approaches for the prevention of osteoporosis [4-8]. Although several studies have reported protective factors for BMD in middle-aged women, these findings remain controversial. Wolff et al. investigated the randomized controlled trials and nonrandomized controlled trials on the effects of exercise training programs on bone in women. As a result, the treatment effects for the nonrandomized controlled trials were almost twice as high as those for the randomized controlled trials [9]. This evidence suggests that exercise is most effective if they are individually tailored and include appropriate types and doses.

Arterial stiffness and Atherosclerosis can be focused as one of the factors of these disagreements. Because recent studies also suggested that there is potential for cross-talk between arterial systems and bone metabolism [10-12]. Atherosclerosis is a chronic inflammatory disease characterised by the accumulation of oxidised lipoproteins in the arterial wall $[13,14]$. Relations between endothelial dysfunction, cholesterol elevation, inflammation, thrombosis and atherogenesis have been established [15]. For example, arterial stiffness and Atherosclerosis have been implicated as a potential contributor to bone health due to the potential effects of nitric oxide (NO) [16-20] and atherogenic materials (e.g., oxidative stress) [21,22] on osteocyte function. Therefore, reducing arterial stiffness may also influence bone health in women with progressing arteriosclerosis.

Physical activity and nutrient intake are modifiable factors that influence arterial stiffness. Habitual physical activity has a positive effect on arterial distensibility [19,23-27]. Boyle et al. provided evidence for the deleterious impact of short-term reductions in daily activity on vascular health [25], as well as evidence that arterial stiffness increases with age in sedentary women. Significant age-related increases in arterial stiffness, however, are not observed in women with high levels of physical activity [23]. Light physical activity has also been shown to be associated with attenuation of arterial stiffening in sedentary individuals [26].

Kahwati et al. reported that neither vitamin D, calcium, nor combined supplementation was associated with reduced fracture incidence in healthy adults without known nutrient deficiency [4]. Avenell et al. evaluated vitamin D for preventing fractures and reported that vitamin $\mathrm{D}$ was beneficial in populations at high-risk of fracture but not low-risk populations [28]. These studies indicated that nutrients may have effects only in specific populations. Therefore, it is important to assess the differences in nutritional effects on BMD in groups with different characteristics. Kruger and Horrobin reported that essential fatty acid-deficient animals develop severe osteoporosis coupled with increased renal and arterial calcification. They suggested that the interaction between essential fatty acids and calcium or vitamin D metabolism may be associated with osteoporosis and ectopic calcification [29]. Many studies have focused on the quantity and type of dietary fat, as well as its effects on arterial stiffness [30-34]. Cross-sectional data suggest that saturated fats adversely affect vascular function, whereas polyunsaturated fatty acids (PUFAs) have beneficial effects [33]. Hu et al. suggested that dietary strategies are effective in preventing arterial disease if they include a substitution of saturated fatty acids and increased consumption of unsaturated fatty acids from fish or plant sources [30]. Physical activity and nutrient intake are considered to be modifiable factors that influence arterial stiffness.

There is limited consensus, however, on the effects of physical activity and nutrient intake on individuals with high or low levels of arterial stiffness. The effects of these types of physical activity and nutrition intake on BMD remain unclear. This pilot study was performed to investigate whether physical activity and nutrient intake are associated with BMD in middle-aged women with high levels of arterial stiffness. 


\section{Materials and Methods}

\subsection{Subjects}

Middle-aged women younger than 65 years of age were recruited from local communities for this study. Data related to sociodemographic characteristics (date of birth), medical history (hypertension, hyperlipidaemia, diabetes mellitus, stroke, heart disease, renal failure and arthralgia), current smoking status and alcohol intake and current medications (name and dosage) were collected during the screening visit. Women with any medical considerations, including taking specific drugs or supplements (osteoporosis, hypertension, hyperlipidaemia, diabetes mellitus, stroke, heart disease, renal failure, arthralgia and history of fracture in the previous 12 months) that could affect bone metabolism were excluded. The study population included seven subjects with SBP $>140 \mathrm{mmHg}$, but they had not received medical treatment. Ten women fulfilled the exclusion criteria. All subjects provided written informed consent after being informed of the benefits and risks of the investigation. This study was approved by the Ethics Committee of Ritsumeikan University (Approval Number: BKC-IRB-2012-032). All participants reviewed and signed informed consent forms in accordance with the Declaration of Helsinki.

\subsection{Dual-Energy X-Ray Absorptiometry}

Body composition was assessed by dual-energy X-ray absorptiometry (DXA) using enCORE software (Lunar Prodigy; GE Healthcare, Buckinghamshire, UK). Subjects fasted overnight and did not perform any exercise in the morning before measurements were taken. In this study, BMD values were derived from total-body DXA scans. Total-body BMD is not an appropriate surrogate for diagnosing osteoporosis or assessing fracture risk [35] because the relationship between total-body BMD and fracture risk has not been adequately defined. On the other hand, Looker et al. suggested that these data provide a unique opportunity to assess differences in BMD between groups across the entire skeleton [36], and it may be useful when investigating the effects of nutrient intake and physical activity, such as aerobic exercise or walking. Total-body and regional BMD, fat mass and fat-free mass (FFM) were analysed. All scans were performed and analysed by a single trained and licensed technician who was blinded to participant group allocation. Body mass index (BMI) was determined by dividing body weight in kilograms $(\mathrm{kg})$ by height in meters squared $\left(\mathrm{m}^{2}\right)$. Skeletal muscle mass index was calculated by dividing the appendicular fat-free mass (FFM) in kilograms $(\mathrm{kg})$ by height in meters squared $\left(\mathrm{m}^{2}\right)$.

\subsection{Measurement of Arterial Stiffness and Blood Pressure}

Pulse wave velocity (PWV) is considered to be an indirect indicator of arterial stiffness, reflecting vascular damage. Subjects rested for 15 minutes in the supine position before carotid-femoral PWV (cf-PWV), brachial-ankle PWV (ba-PWV) and blood pressure measurements were taken. The cf-PWV, ba-PWV and blood pressure were measured simultaneously using a vascular testing device (form PWV/Ankle brachial index (ABI); Omron Colin, Kyoto, Japan). The cf-PWV was measured using applanation tonometry, with an array of 15 transducers (form PWV/ABI), as described previously [37-39]. The distance travelled by each pulse wave was assessed by a random zero-length measurement on the surface of the body using a non-elastic tape measure. Pulse wave transit time was determined by measuring the time delay between the proximal and distal foot waveforms. The foot of the wave was identified as the commencement of the sharp systolic upstroke, which was detected automatically. In this study, the coefficient of variation for interobserver reproducibility of cf-PWV was $4.7 \%$. The mean values of systolic and diastolic blood pressure in the right and left arms were calculated for analysis.

\subsection{Analysis of Blood Samples}

Blood was drawn from subjects in the seated position. Fasting ( $>12 \mathrm{~h})$ blood samples were collected by venipuncture in tubes with or without ethylene diamine tetraacetic acid (for plasma or serum). Blood samples were centrifuged at $1500 \mathrm{rpm}$ for $15 \mathrm{~min}$ and were stored at $-20^{\circ} \mathrm{C}$. The serum 
concentration of triglycerides was determined using commercial kits (Mitsubishi Chemical Medience, Tokyo, Japan). Serum high-density lipoprotein cholesterol (HDL-C) was measured by an enzymatic method (Mitsubishi Chemical Medience). Fasting plasma glucose (FPG) was measured by the glucose dehydrogenase method. Whole-blood glycohemoglobin A1c (HbA1c) was measured by an enzymatic method (Glycohemoglobin A1c kit; Mitsubishi Chemical Medience).

\subsection{Evaluation of Physical Activity}

The duration and intensity of physical activity in free-living conditions were evaluated using a triaxial accelerometer (Active Style Pro HJA-350IT; Omron Healthcare; Muko, Kyoto, Japan) [40,41]. The accelerometer was attached to the waist of each subject during waking hours for 7 days, including both weekdays and weekends. Valid data were those that included at least 10 hours of wearing time. The criterion for acceptable pedometer data was that data were collected at least three days per week, including at least one weekday and one weekend day. The Omron Active Style Pro HJA-350IT measures activity intensity over a 60-s period and estimates the metabolic equivalents of the task (METs). Steps and total minutes spent in moderate to vigorous physical activity (MVPA; $\geq 3$ METs) taken per day were used as physical activity outcome measures.

\subsection{Nutrient Intake}

Nutrient intake was measured using the brief-type self-administered diet history questionnaire (BDHQ), which is a fixed-portion questionnaire that assesses dietary intake during the previous month $[42,43]$. The unadjusted intakes of energy and nutrients measured by the BDHQ were calculated using an ad hoc computer algorithm based on the Standard Tables of Food Composition in Japan. Intakes of saturated fatty acids, monounsaturated fatty acids (MUFAs), PUFAs, $n-3$ fatty acids and n-6 fatty acids were calculated in grams per day using the food list section of the BDHQ. Total energy was calculated in kilocalories per day from BDHQ responses. Questionnaires indicating an extreme energy intake of greater than two standard deviations were considered to be invalid and were excluded.

\subsection{Other Variables}

Covariates were measured at the same time as the DXA assessment and included age (years) and height $(\mathrm{cm})$. Height without shoes was measured using a stadiometer.

\subsection{Statistical Analysis}

Outcome measures included BMD (arm, spine, pelvis, leg and total body), physical activity and nutritional intake. Fat intake was defined as SFA, MUFA and PUFA in grams per day. Fat intake was then adjusted for total energy intake with the residual method [44]. In this method, energy-adjusted nutrient intakes are computed as the residuals from the regression model with total caloric intake as the independent variable and absolute nutrient intake as the dependent variable. Potential differences between the Low- and High-PWV groups were assessed using the independent $t$-test. All measurements and calculated values are expressed as the means \pm standard deviation. Correlation analyses of total-body and regional BMD with study variables were performed using Pearson's correlation analyses. Partial correlations were adjusted for age and BMI and used to examine the relationships between BMD, physical activity and nutritional intake. All analyses were performed using SPSS (version 19; IBM, Chicago, IL, USA). Statistical significance was set at $\alpha<0.05$.

\section{Results}

We assessed cf-PWV to evaluate arterial stiffness (median cf-PWV $=1053 \mathrm{~cm} / \mathrm{s}$ ). All subjects were classified into two groups by cf-PWV (Low- and High-PWV groups). Descriptive characteristics for Low- and High-PWV groups, as well as all subjects, are shown in Tables 1-3. Clinical features were similar for Low- and High-PWV groups, except for arterial stiffness parameters (Table 1). Findings 
regarding physical activity and nutrient intake were similar in both Low- and High-PWV groups, except for SFA intake (Tables 2 and 3).

Table 1. Subject characteristics at baseline for the whole study group and for Low- and High-PWV groups separately.

\begin{tabular}{|c|c|c|c|c|}
\hline \multirow[b]{2}{*}{ Varieties } & All $(n=22)$ & Low $(n=11)$ & High $(n=11)$ & \multirow[b]{2}{*}{$p$} \\
\hline & Mean \pm SD & Mean \pm SD & Mean \pm SD & \\
\hline Age (yrs) & $55.1 \pm 6.2$ & $55.4 \pm 5.4$ & $54.8 \pm 7.1$ & 0.84 \\
\hline $\operatorname{BMI}\left(\mathrm{kg} / \mathrm{m}^{2}\right)$ & $20.0 \pm 1.9$ & $20.2 \pm 1.7$ & $19.8 \pm 2.1$ & 0.57 \\
\hline Body fat percentage (\%) & $26.4 \pm 6.0$ & $27.2 \pm 4.7$ & $25.7 \pm 7.3$ & 0.58 \\
\hline $\mathrm{SMI}\left(\mathrm{kg} / \mathrm{m}^{2}\right)$ & $5.7 \pm 0.5$ & $5.8 \pm 0.3$ & $5.7 \pm 0.7$ & 0.60 \\
\hline \multicolumn{5}{|l|}{$\operatorname{BMD}\left(\mathrm{g} / \mathrm{cm}^{2}\right)$} \\
\hline Total body & $1.036 \pm 0.107$ & $1.078 \pm 0.103$ & $0.994 \pm 0.099$ & 0.07 \\
\hline Arm & $0.734 \pm 0.068$ & $0.745 \pm 0.061$ & $0.722 \pm 0.075$ & 0.44 \\
\hline Spine & $0.941 \pm 0.149$ & $0.979 \pm 0.155$ & $0.903 \pm 0.140$ & 0.24 \\
\hline Pelvis & $0.989 \pm 0.128$ & $1.034 \pm 0.106$ & $0.945 \pm 0.138$ & 0.10 \\
\hline Leg & $1.083 \pm 0.122$ & $1.132 \pm 0.103$ & $1.033 \pm 0.124$ & 0.06 \\
\hline \multicolumn{5}{|l|}{ Atherosclerosis parameter } \\
\hline cf-PWV $(\mathrm{cm} / \mathrm{s})$ & $1071 \pm 145$ & $953 \pm 70$ & $1189 \pm 94$ & $<0.01 * *$ \\
\hline ba-PWV (cm/s) & $1472 \pm 375$ & $1251 \pm 219$ & $1693 \pm 374$ & $<0.01 * *$ \\
\hline $\mathrm{SBP}(\mathrm{mmHg})$ & $126 \pm 25$ & $111 \pm 17$ & $142 \pm 22$ & $<0.01 * *$ \\
\hline $\mathrm{DBP}(\mathrm{mmHg})$ & $75 \pm 14$ & $67 \pm 11$ & $82 \pm 12$ & $<0.01 * *$ \\
\hline \multicolumn{5}{|l|}{ Biochemical parameter } \\
\hline Total cholesterol (mg/dl) & $228 \pm 37$ & $221 \pm 38$ & $235 \pm 36$ & 0.38 \\
\hline Triglyceride (mg/dl) & $77 \pm 35$ & $74 \pm 30$ & $80 \pm 41$ & 0.66 \\
\hline HDL cholesterol (mg/dl) & $87 \pm 20$ & $86 \pm 21$ & $88 \pm 19$ & 0.87 \\
\hline LDL cholesterol (mg/dl) & $125 \pm 35$ & $120 \pm 37$ & $131 \pm 34$ & 0.46 \\
\hline HDL percentage (\%) & $39 \pm 10$ & $40 \pm 10$ & $38 \pm 9$ & 0.68 \\
\hline Blood sugar (mg/dl) & $88 \pm 6$ & $87 \pm 4$ & $89 \pm 8$ & 0.39 \\
\hline $\mathrm{HbA1c}(\%)$ & $5.1 \pm 0.3$ & $5.0 \pm 0.3$ & $5.2 \pm 0.4$ & 0.24 \\
\hline
\end{tabular}

Data are presented as means \pm SD. BMI, body mass index; SMI, skeletal mass index; BMD, bone mineral density; SBP, systolic blood pressure; DBP, diastolic blood pressure; cf-PWV, carotid-femoral pulse wave velocity; ba-PWV, brachial-ankle pulse wave velocity. Potential differences between the Low- and High-PWV groups were assessed using the independent t-test. ${ }^{* *}, p<0.01$.

Table 2. Physical activity parameters for the whole study group and Low- and High-PWV groups separately.

\begin{tabular}{ccccc}
\hline \multirow{2}{*}{ Varieties } & All $(\mathbf{n}=\mathbf{2 2})$ & Low $(\mathbf{n}=\mathbf{1 1})$ & High $(\mathbf{n = 1 1})$ & \multirow{2}{*}{$\boldsymbol{p}$} \\
\cline { 2 - 4 } & Mean \pm SD & Mean \pm SD & Mean \pm SD & \\
\hline Accelerometry parameter (/day) & & & & \\
Total MVPA (MET * hour) & $5.1 \pm 2.4$ & $5.7 \pm 2.6$ & $4.6 \pm 2.1$ & 0.30 \\
Locomotive & $2.0 \pm 0.8$ & $2.1 \pm 0.7$ & $1.9 \pm 1.0$ & 0.47 \\
Non-Locomotive & $3.1 \pm 2.2$ & $3.6 \pm 2.6$ & $2.7 \pm 1.8$ & 0.37 \\
Steps & $7349 \pm 1663$ & $7467 \pm 1238$ & $7231 \pm 2061$ & 0.75 \\
\hline
\end{tabular}

Data are presented as means \pm SD. MVPA, moderate-to-vigorous physical activity. Potential differences between the Low- and High-PWV groups were assessed using the independent $\mathrm{t}$-test. ${ }^{*}, p<0.05$. 
Table 3. Nutritional parameters for the whole study group and Low- and High-PWV groups separately.

\begin{tabular}{|c|c|c|c|c|}
\hline \multirow[b]{2}{*}{ Varieties } & All $(n=22)$ & Low $(n=9)$ & High $(n=11)$ & \multirow[b]{2}{*}{$p$} \\
\hline & Mean \pm SD & Mean \pm SD & Mean \pm SD & \\
\hline \multicolumn{5}{|l|}{ Nutritional parameters (/day) } \\
\hline Protein $(g)$ & $68.6 \pm 11.3$ & $70.4 \pm 14.9$ & $67.2 \pm 8.1$ & 0.55 \\
\hline Fat $(\mathrm{g})$ & $53.5 \pm 8.5$ & $50.9 \pm 8.5$ & $55.7 \pm 7.0$ & 0.18 \\
\hline SFA & $14.5 \pm 3.7$ & $12.7 \pm 2.8$ & $15.9 \pm 3.3$ & $0.03 *$ \\
\hline MUFA & $19.1 \pm 3.5$ & $18.2 \pm 4.1$ & $19.8 \pm 2.5$ & 0.29 \\
\hline PUFA & $12.7 \pm 2.2$ & $12.9 \pm 2.2$ & $12.6 \pm 2.2$ & 0.75 \\
\hline n-3 fatty acids & $2.6 \pm 0.6$ & $2.8 \pm 0.7$ & $2.4 \pm 0.5$ & 0.21 \\
\hline n- 6 fatty acids & $10.1 \pm 1.9$ & $10.1 \pm 1.9$ & $10.1 \pm 1.8$ & 0.96 \\
\hline Carbohydrate (g) & $238 \pm 22$ & $242 \pm 28$ & $234 \pm 17$ & 0.45 \\
\hline Sodium (mg) & $3737 \pm 606$ & $3915 \pm 673$ & $3592 \pm 529$ & 0.24 \\
\hline Potassium (mg) & $2864 \pm 711$ & $3011 \pm 925$ & $2744 \pm 509$ & 0.42 \\
\hline Calcium (mg) & $564 \pm 148$ & $580 \pm 194$ & $550 \pm 105$ & 0.67 \\
\hline Magnesium (mg) & $261 \pm 62$ & $278 \pm 81$ & $246 \pm 40$ & 0.26 \\
\hline Phosphorus (mg) & $1064 \pm 201$ & $1103 \pm 268$ & $1031 \pm 137$ & 0.45 \\
\hline Iron (mg) & $8.3 \pm 2.1$ & $9.0 \pm 2.5$ & $7.7 \pm 1.5$ & 0.18 \\
\hline Zinc (mg) & $8.1 \pm 0.9$ & $8.5 \pm 1.0$ & $7.9 \pm 0.8$ & 0.17 \\
\hline Copper (mg) & $1.2 \pm 0.2$ & $1.2 \pm 0.2$ & $1.1 \pm 0.2$ & 0.10 \\
\hline Manganese (mg) & $3.5 \pm 0.8$ & $3.5 \pm 0.8$ & $3.4 \pm 0.9$ & 0.80 \\
\hline$\beta$-carotene eq (mg) & $4842 \pm 2377$ & $5739 \pm 3025$ & $4107 \pm 1532$ & 0.13 \\
\hline Vitamin D $(\mu \mathrm{g})$ & $14.2 \pm 8.7$ & $17 \pm 12$ & $12 \pm 4$ & 0.24 \\
\hline$\alpha$-tocopherol eq (mg) & $9.3 \pm 1.9$ & $9.7 \pm 2.1$ & $9.0 \pm 1.7$ & 0.41 \\
\hline Vitamin $K(\mu \mathrm{g})$ & $324 \pm 154$ & $382 \pm 168$ & $277 \pm 132$ & 0.13 \\
\hline Vitamin B1 (mg) & $0.8 \pm 0.1$ & $0.9 \pm 0.2$ & $0.8 \pm 0.1$ & 0.68 \\
\hline Vitamin B2 (mg) & $1.4 \pm 0.3$ & $1.4 \pm 0.4$ & $1.4 \pm 0.3$ & 0.93 \\
\hline Niacin (mg) & $17.3 \pm 4.1$ & $18.0 \pm 5.4$ & $16.7 \pm 2.7$ & 0.49 \\
\hline Vitamin B6 (mg) & $1.4 \pm 0.3$ & $1.5 \pm 0.4$ & $1.3 \pm 0.3$ & 0.27 \\
\hline Vitamin B12 ( $\mu \mathrm{g})$ & $8.8 \pm 4.3$ & $9.9 \pm 5.6$ & $7.8 \pm 2.6$ & 0.29 \\
\hline Folic acid $(\mu \mathrm{g})$ & $401 \pm 130$ & $444 \pm 158$ & $366 \pm 99$ & 0.19 \\
\hline Pantothenic acid (mg) & $6.7 \pm 1.2$ & $6.9 \pm 1.5$ & $6.5 \pm 1.0$ & 0.56 \\
\hline Vitamin C (mg) & $146 \pm 57$ & $158 \pm 69$ & $136 \pm 46$ & 0.41 \\
\hline Suger (g) & $13.4 \pm 6.6$ & $11.0 \pm 6.1$ & $15.3 \pm 4.2$ & 0.08 \\
\hline Alcohol (g) & $5.4 \pm 10.4$ & $10.8 \pm 12.8$ & $0.9 \pm 1.4$ & 0.02 \\
\hline Genistein (mg) & $20.8 \pm 14.2$ & $24.7 \pm 14.5$ & $17.6 \pm 13.6$ & 0.27 \\
\hline
\end{tabular}

Data are presented as means \pm SD. SFA, saturated fatty acid; MUFA, onounsaturated fatty acid; PUFA, polyunsaturated fatty acid; Eq, equivalents. Potential differences between the Low- and High-PWV groups were assessed using the independent t-test. ${ }^{*}, p<0.05$.

Table 4 summarises the results of correlation analysis regarding the relationships between clinical features and BMD. Total-body, arm and leg BMD were negatively associated with age in all subjects $(\mathrm{r}=-0.44, \mathrm{r}=-0.60, \mathrm{r}=-0.51$, respectively). Similar results were observed in the High-PWV group. In all subjects, the spine and pelvis BMD were associated with BMI $(r=0.46, r=0.49$, respectively).

In this study, age was associated with total and non-locomotive MVPA in all subjects $(r=0.49$, $r=0.46$, respectively) and BMI was associated with locomotive MVPA $(r=0.52)$. Similar results were observed in the High-PWV group. BMI and body fat were associated with total MVPA ( $r=0.66$, $r=0.68$, respectively). In the Low-PWV group, age was associated with total and non-locomotive MVPA $(r=0.64, r=0.63$, respectively, $p<0.05)$. To exclude the possible effect of age on physical activity, we performed partial correlation analysis.

In the High-PWV group, using a partial correlation model, the number of steps taken showed a significant association with leg BMD $(r=0.67)$ when adjusted for age and BMI (Table 5). Significant associations were observed between MUFA and total-body, spine and pelvis BMD $(r=0.82, r=0.88$, $\mathrm{r}=0.72$, respectively). Similarly, PUFA results were associated with total-body, arm, spine and pelvis $\operatorname{BMD}(r=0.80, r=0.77, r=0.88, r=0.74$, respectively). Moreover, $\alpha$-tocopherol equivalents (Eq) results were associated with total-body, arm, and spine $\operatorname{BMD}(r=0.68, r=0.71, r=0.72$, respectively). 
Table 4. Correlation coefficients between analysed characteristics variables and bone mineral density.

\begin{tabular}{|c|c|c|c|c|c|c|c|c|c|c|c|c|c|c|c|}
\hline \multirow[b]{2}{*}{ Varieties } & \multicolumn{5}{|c|}{ All $(n=22)$} & \multicolumn{5}{|c|}{ Low $(\mathrm{n}=11)$} & \multicolumn{5}{|c|}{ High (n=11) } \\
\hline & ТВ & Arm & Spine & Pelvis & Leg & ТВ & Arm & Spine & Pelvis & Leg & TB & Arm & Spine & Pelvis & Leg \\
\hline Age & $-0.44 *$ & $-0.60 * *$ & -0.28 & -0.37 & $-0.51 *$ & -0.21 & -0.33 & -0.11 & -0.41 & -0.31 & $-0.74^{* *}$ & $-0.79 * *$ & -0.47 & -0.42 & $-0.75 * *$ \\
\hline BMI & 0.39 & 0.37 & 0.46 * & $0.49 *$ & 0.42 & 0.34 & 0.18 & 0.33 & 0.30 & 0.42 & 0.41 & 0.48 & 0.57 & 0.59 & 0.39 \\
\hline SMI & 0.12 & 0.16 & -0.03 & -0.04 & 0.34 & 0.16 & 0.05 & -0.09 & -0.12 & 0.18 & 0.06 & 0.19 & -0.05 & -0.08 & 0.15 \\
\hline Body fat & 0.27 & 0.23 & 0.42 & $0.52 *$ & 0.18 & 0.31 & 0.23 & 0.40 & 0.40 & 0.44 & 0.20 & 0.21 & 0.42 & 0.56 & 0.25 \\
\hline \multicolumn{16}{|c|}{ Atherosclerosis parameter } \\
\hline cf-PWV & -0.23 & -0.03 & -0.03 & -0.14 & -0.34 & -0.01 & 0.18 & 0.22 & 0.41 & 0.11 & 0.37 & 0.24 & 0.47 & 0.24 & -0.04 \\
\hline ba-PWV & -0.02 & 0.11 & 0.29 & 0.14 & -0.20 & 0.33 & 0.38 & 0.58 & 0.54 & 0.32 & 0.30 & 0.24 & 0.63 & 0.46 & -0.05 \\
\hline SBP & -0.10 & -0.01 & 0.11 & -0.14 & -0.28 & 0.28 & 0.37 & 0.57 & 0.46 & 0.33 & 0.17 & 0.00 & 0.23 & -0.07 & -0.23 \\
\hline \multirow{2}{*}{\multicolumn{16}{|c|}{ Biochemical parameter }} \\
\hline & & & & & & & & & & & & & & & \\
\hline Total cholesterol & 0.17 & 0.26 & $0.44^{*}$ & 0.39 & 0.14 & 0.23 & 0.34 & 0.51 & 0.23 & 0.21 & 0.32 & 0.29 & 0.54 & $0.73 *$ & 0.28 \\
\hline Triglyceride & 0.09 & 0.09 & 0.21 & 0.29 & 0.12 & -0.34 & -0.47 & -0.26 & -0.29 & -0.27 & 0.50 & 0.46 & $0.66^{*}$ & $0.71 *$ & 0.46 \\
\hline HDL cholesterol & -0.09 & 0.03 & $\begin{array}{l}.0 .13 \\
-0.13\end{array}$ & -0.18 & -0.03 & -0.26 & -0.04 & -0.26 & -0.38 & -0.19 & 0.11 & 0.10 & 0.04 & -0.02 & 0.13 \\
\hline LDL cholesterol & 0.21 & 0.24 & 0.50 * & $0.45^{*}$ & 0.14 & 0.44 & 0.45 & $0.72 *$ & 0.50 & 0.37 & 0.15 & 0.13 & 0.39 & $0.60^{*}$ & 0.11 \\
\hline $\begin{array}{l}\text { HDL percentage } \\
\text { HDL pertat }\end{array}$ & $\begin{array}{l}0.21 \\
-0.14\end{array}$ & $\begin{array}{l}0.24 \\
-0.09\end{array}$ & $\begin{array}{l}0.00 \\
-0.35\end{array}$ & $\begin{array}{l}0.45 \\
-0.34\end{array}$ & $\begin{array}{l}0.14 \\
-0.08\end{array}$ & $\begin{array}{c}0.44 \\
-0.31\end{array}$ & $\begin{array}{l}0.45 \\
-0.17\end{array}$ & $\begin{array}{l}0.12 \\
-0.50\end{array}$ & $\begin{array}{l}0.00 \\
-0.40\end{array}$ & $\begin{array}{l}0.02 \\
-0.25\end{array}$ & $\begin{array}{l}-0.13 \\
-0.07\end{array}$ & $\begin{array}{l}0.13 \\
-0.05\end{array}$ & $\begin{array}{l}0.39 \\
-0.26\end{array}$ & $\begin{array}{l}0.00 \\
-0.41\end{array}$ & $\begin{array}{l}0.11 \\
-0.02\end{array}$ \\
\hline Blood sugar & $\begin{array}{l}-0.14 \\
-0.27\end{array}$ & $\begin{array}{l}-0.099 \\
-0.15\end{array}$ & $\begin{array}{l}-0.53 \\
-0.18\end{array}$ & $\begin{array}{l}-0.54 \\
-0.21\end{array}$ & $\begin{array}{l}-0.08 \\
-0.16\end{array}$ & $\begin{array}{c}-0.31 \\
0.00\end{array}$ & $\begin{array}{l}-0.11 \\
-0.04\end{array}$ & $\begin{array}{l}-0.00 \\
-0.03\end{array}$ & $\begin{array}{l}-0.40 \\
-0.19\end{array}$ & $\begin{array}{c}-0.23 \\
0.12\end{array}$ & $\begin{array}{l}-0.07 \\
-0.35\end{array}$ & $\begin{array}{l}-0.03 \\
-0.16\end{array}$ & $\begin{array}{l}-0.26 \\
-0.21\end{array}$ & $\begin{array}{l}-0.41 \\
-0.15\end{array}$ & $\begin{array}{l}-0.02 \\
-0.18\end{array}$ \\
\hline \multirow{2}{*}{\multicolumn{16}{|c|}{$\begin{array}{l}\text { Accelerometry parameter } \\
\text { A. }\end{array}$}} \\
\hline & & & & & & & & & & & & & & & \\
\hline Total MVPA & -0.19 & -0.15 & 0.00 & 0.04 & -0.03 & -0.52 & -0.40 & -0.29 & -0.50 & -0.39 & -0.06 & -0.01 & 0.24 & 0.39 & .11 \\
\hline Locomotive & 0.10 & 0.18 & 0.11 & 0.18 & 0.34 & 0.39 & 0.43 & 0.47 & 0.40 & 0.55 & -0.22 & -0.01 & -0.25 & -0.02 & 0.15 \\
\hline Non-Locomotive & -0.24 & -0.23 & -0.04 & -0.02 & -0.16 & $-0.64 *$ & -0.54 & -0.43 & $-0.63 *$ & -0.56 & 0.05 & 0.00 & 0.42 & 0.48 & 0.04 \\
\hline Steps & -0.01 & -0.01 & 0.01 & 0.10 & 0.20 & 0.20 & 0.03 & 0.34 & 0.22 & 0.15 & -0.20 & -0.06 & -0.24 & 0.02 & 0.22 \\
\hline \multicolumn{16}{|c|}{$\begin{array}{l}\text { Sutritionsal parameters } \\
\mathrm{a}^{\mathrm{a}}\end{array}$} \\
\hline Protein & -0.15 & -0.26 & -0.15 & -0.15 & -0.08 & 0.02 & -0.18 & -0.15 & -0.15 & 0.08 & -0.51 & -0.42 & -0.15 & -0.32 & -0.37 \\
\hline Fat & 0.21 & 0.28 & 0.18 & 0.18 & 0.18 & -0.10 & -0.11 & 0.18 & 0.18 & -0.17 & 0.53 & 0.35 & 0.18 & 0.45 & 0.39 \\
\hline SFA & 0.15 & 0.27 & 0.11 & 0.11 & 0.15 & -0.08 & -0.04 & 0.11 & 0.11 & -0.17 & 0.42 & 0.28 & 0.11 & 0.35 & 0.37 \\
\hline MUFA & 0.22 & 0.31 & 0.21 & 0.21 & 0.19 & -0.10 & -0.04 & 0.21 & 0.21 & -0.13 & 0.63 & 0.47 & 0.21 & 0.55 & 0.45 \\
\hline PUFA & 0.20 & 0.11 & 0.18 & 0.18 & 0.12 & -0.07 & -0.18 & 0.18 & 0.18 & -0.15 & 0.34 & 0.21 & 0.18 & 0.31 & 0.18 \\
\hline $\mathrm{n}-3$ fatty acids & 0.09 & -0.14 & 0.07 & 0.07 & -0.01 & 0.15 & -0.13 & 0.07 & 0.07 & 0.05 & 0.01 & -0.05 & 0.07 & -0.02 & -0.06 \\
\hline $\mathrm{n}-6$ fatty acids & 0.20 & 0.18 & 0.18 & 0.18 & 0.14 & -0.14 & -0.16 & 0.18 & 0.18 & -0.19 & 0.41 & 0.27 & 0.18 & 0.38 & 0.24 \\
\hline Carbohydrate & -0.04 & 0.10 & -0.01 & -0.01 & 0.03 & 0.00 & 0.15 & -0.01 & -0.01 & 0.07 & -0.24 & -0.11 & -0.01 & -0.23 & -0.17 \\
\hline Sodium & -0.21 & -0.38 & -0.16 & -0.16 & -0.21 & 0.02 & -0.23 & -0.16 & -0.16 & 0.03 & -0.47 & -0.39 & -0.16 & -0.33 & -0.39 \\
\hline Potassium & 0.16 & 0.06 & 0.12 & 0.12 & 0.20 & 0.25 & 0.09 & 0.12 & 0.12 & 0.37 & -0.12 & -0.08 & 0.12 & -0.02 & -0.15 \\
\hline Calcium & 0.15 & 0.01 & 0.10 & 0.10 & 0.20 & 0.23 & 0.03 & 0.10 & 0.10 & 0.28 & -0.05 & -0.10 & 0.10 & -0.01 & 0.03 \\
\hline Magnesium & 0.00 & -0.14 & -0.02 & -0.02 & 0.05 & 0.17 & 0.00 & -0.02 & -0.02 & 0.28 & -0.39 & -0.32 & -0.02 & -0.24 & -0.34 \\
\hline Phosphorus & -0.15 & -0.26 & -0.15 & -0.15 & -0.08 & 0.02 & -0.18 & -0.15 & -0.15 & 0.08 & -0.51 & -0.42 & -0.15 & -0.32 & -0.37 \\
\hline Iron & -0.13 & -0.27 & -0.18 & -0.18 & -0.05 & -0.05 & -0.23 & -0.18 & -0.18 & 0.06 & -0.52 & -0.42 & -0.18 & -0.41 & -0.41 \\
\hline Zinc & -0.27 & -0.31 & -0.18 & -0.18 & -0.15 & -0.18 & -0.26 & -0.18 & -0.18 & 0.01 & -0.46 & -0.32 & -0.18 & -0.17 & -0.35 \\
\hline Copper & -0.15 & -0.27 & -0.15 & -0.15 & -0.10 & -0.01 & -0.17 & -0.15 & -0.15 & 0.10 & -0.48 & -0.37 & -0.15 & -0.30 & -0.41 \\
\hline Manganese & -0.51 * & $-0.57 * *$ & $-0.48^{* *}$ & -0.48 * & -0.43 & -0.18 & -0.46 & -0.48 & -0.48 & -0.29 & $-0.74 * *$ & -0.58 & -0.48 & $-0.65^{*}$ & -0.48 \\
\hline$\beta$-carotene eq & 0.19 & 0.10 & 0.13 & 0.13 & 0.26 & 0.09 & -0.01 & 0.13 & 0.1 & 0.30 & 0.00 & 0.06 & 0.13 & -0.02 & -0.11 \\
\hline Vitamin D & -0.02 & $\begin{array}{l}0.10 \\
-0.22\end{array}$ & $\begin{array}{l}.0 .04 \\
-0.10\end{array}$ & -0.04 & -0.01 & 0.17 & -0.06 & -0.04 & -0.04 & 0.18 & $-0.61 *$ & $\begin{array}{l}-0.40 \\
-0.49\end{array}$ & -0.04 & $\begin{array}{l}-0.02 \\
-0.52\end{array}$ & $\begin{array}{l}-0.11 \\
-0.46\end{array}$ \\
\hline$\alpha$-tocopherol eq & $\begin{array}{c}-0.02 \\
0.30\end{array}$ & $\begin{array}{l}-0.21 \\
0.21\end{array}$ & $\begin{array}{l}-0.04 \\
0.19\end{array}$ & 0.19 & 0.33 & 0.05 & $\begin{array}{l}-0.00 \\
-0.06\end{array}$ & $\begin{array}{l}-0.04 \\
0.19\end{array}$ & 0.1 & 0.21 & $\begin{aligned}-0.01 \\
0.27\end{aligned}$ & 0. & 0. & 0.15 & $\begin{array}{l}-0.46 \\
0.13\end{array}$ \\
\hline Vitamin K & 0.0 & $\begin{array}{l}0.21 \\
-0.04\end{array}$ & $\begin{array}{l}.0 .019 \\
-0.01\end{array}$ & -0.01 & 0.1 & $\begin{array}{l}0.03 \\
-0.02\end{array}$ & $\begin{array}{l}-0.010 \\
-0.16\end{array}$ & -0.01 & $\begin{array}{ll}0.1 \\
-0 .\end{array}$ & 0. & $\begin{array}{l}-0.21 \\
-0.21\end{array}$ & $\begin{array}{l}0.10 \\
-0.13\end{array}$ & -0.01 & -0.13 & $\begin{array}{l}.10 \\
-0.20\end{array}$ \\
\hline Vitamin & 0.02 & 0.00 & 0.02 & 0.02 & 0.09 & 0.11 & 0. & 0.02 & 0.0 & 0.35 & -0.04 & 0. & 0.02 & 0.07 & -0.10 \\
\hline Vitamin B2 & -0.17 & -0.28 & -0.20 & -0.20 & -0.09 & -0.12 & -0.34 & -0.20 & -0 & -0.12 & -0 & -0.34 & -0.20 & -0.25 & -0.20 \\
\hline $\begin{array}{l}\text { Nitamin b2 } \\
\text { Niacin }\end{array}$ & $\begin{array}{l}-0.17 \\
-0.06\end{array}$ & $\begin{array}{l}-0.28 \\
-0.20\end{array}$ & $\begin{array}{l}-0.20 \\
-0.07\end{array}$ & $\begin{array}{l}-0.20 \\
-0.07\end{array}$ & $\begin{array}{l}-0.09 \\
-0.04\end{array}$ & $\begin{array}{l}-0.12 \\
0.10\end{array}$ & $\begin{array}{l}-0.04 \\
-0.13\end{array}$ & $\begin{array}{l}-0.20 \\
-0.07\end{array}$ & $\begin{array}{l}-0 . \\
-0 .\end{array}$ & 0. & $\begin{array}{l}-0 \\
-0 . \\
-0\end{array}$ & $\begin{array}{l}-0 \\
-0\end{array}$ & $\begin{array}{l}-0.20 \\
-0.07\end{array}$ & $\begin{array}{l}-0.25 \\
-0.26\end{array}$ & $\begin{array}{l}-0.20 \\
-0.34\end{array}$ \\
\hline Vitamin B6 & 0.04 & -0.07 & 0.00 & 0.0 & 0.0 & 0. & -0 & 0.0 & 0.1 & 0.2 & -0.18 & -0.09 & & -0.09 & -0.17 \\
\hline Vitamin & -0 & -0.28 & -0 . & -0 & -0 . & 0. & -0 & -0.12 & -0 & 0. & -0 & -0.36 & -0.12 & -0.40 & -0.34 \\
\hline & 0.8 & -0 & -0 & -0 & & & -0 & -0 & -0 . & 0. & -0 & & -0.01 & -0.36 & -0.30 \\
\hline Pantothenic acid & -0.27 & -0 & -6 & -0 & -0.15 & -0.18 & -0.2 & -0 . & -0 & 0.01 & -0.46 & -0.32 & -0.18 & -0.17 & -0.35 \\
\hline
\end{tabular}


Table 4. Cont.

\begin{tabular}{|c|c|c|c|c|c|c|c|c|c|c|c|c|c|c|c|}
\hline \multirow{2}{*}{ Varieties } & \multicolumn{5}{|c|}{ All $(n=22)$} & \multicolumn{5}{|c|}{ Low $(n=11)$} & \multicolumn{5}{|c|}{ High $(n=11)$} \\
\hline & TB & Arm & Spine & Pelvis & Leg & TB & Arm & Spine & Pelvis & Leg & TB & Arm & Spine & Pelvis & Leg \\
\hline Vitamin C & 0.23 & 0.14 & 0.16 & 0.16 & 0.30 & 0.32 & 0.19 & 0.16 & 0.16 & 0.46 & -0.14 & -0.09 & 0.16 & -0.12 & -0.10 \\
\hline Suger & 0.30 & $0.50^{*}$ & 0.15 & 0.15 & 0.40 & 0.34 & 0.59 & 0.15 & 0.15 & 0.40 & 0.02 & 0.01 & 0.15 & -0.31 & 0.14 \\
\hline Alcohol & -0.02 & -0.18 & -0.04 & -0.04 & -0.09 & 0.13 & 0.07 & -0.04 & -0.04 & 0.09 & 0.16 & 0.07 & -0.04 & -0.18 & 0.10 \\
\hline Genistein & -0.20 & -0.33 & -0.17 & -0.17 & -0.19 & -0.13 & -0.30 & -0.17 & -0.17 & -0.18 & -0.29 & -0.25 & -0.17 & -0.14 & -0.21 \\
\hline
\end{tabular}

TB, total-body; BMI, body mass index; SMI, skeletal mass index; cf-PWV, carotid-femoral pulse wave velocity; ba-PWV, brachial-ankle pulse wave velocity; SBP, systolic blood pressure; DBP, diastolic blood pressure; MVPA, moderate-to-vigorous physical activity; SFA, saturated fatty acid; MUFA, monounsaturated fatty acid; PUFA, polyunsaturated fatty acid; Eq, equivalents. ${ }^{a}$ All, $n=20$; Low-PWV, $n=9$; High-PWV, $n=11$. Correlation analyses of the relationships between BMD and clinical features. ${ }^{*} p<0.05, * *<<0.01$.

Table 5. Partial correlation coefficients adjusted for age and BMI between physical activity, nutritional parameters and bone mineral density.

\begin{tabular}{|c|c|c|c|c|c|c|c|c|c|c|c|c|c|c|c|}
\hline \multirow[b]{2}{*}{ Varieties } & \multicolumn{5}{|c|}{ All $(\mathrm{n}=22)$} & \multicolumn{5}{|c|}{ Low $(\mathrm{n}=11)$} & \multicolumn{5}{|c|}{ High $(n=11)$} \\
\hline & TB & Arm & Spine & Pelvis & Leg & TB & Arm & Spine & Pelvis & Leg & TB & Arm & Spine & Pelvis & Leg \\
\hline Accelerometry param & & & & & & & & & & & & & & & \\
\hline Total MVPA & -0.19 & 0.02 & -0.06 & 0.06 & & -0.59 & -0.29 & -0.35 & -0.40 & -0.37 & -0.05 & 0.04 & 0.12 & 0.36 & \\
\hline Locomotive & 0.00 & 0.22 & -0.09 & 0.02 & 0.38 & 0.29 & 0.52 & 0.38 & 0.41 & 0.49 & -0.39 & 0.00 & -0.63 & -0.29 & 0.41 \\
\hline Non-Locomotive & -0.18 & -0.06 & -0.02 & 0.06 & -0.03 & $-0.69 *$ & -0.44 & -0.46 & -0.52 & -0.51 & 0.20 & 0.04 & 0.50 & 0.53 & 0.21 \\
\hline & & & -0.12 & 0.04 & & & & & & -0.08 & -0.12 & 0.18 & -0.37 & & 0.67 \\
\hline \multicolumn{16}{|c|}{ Nutritional parameters ${ }^{a}$} \\
\hline $\begin{array}{l}\text { Protein } \\
\text { Fat }\end{array}$ & 0.05 & 0.02 & -0.16 & 0.03 & 0.21 & $\begin{array}{l}0.06 \\
-0.15\end{array}$ & -0.06 & -0.18 & -0.06 & $\begin{array}{c}0.20 \\
-0.25\end{array}-1$ & -0.09 & $\begin{array}{l}0.24 \\
0.58\end{array}$ & -0.19 & 0.25 & 0.23 \\
\hline $\begin{array}{l}\text { Fat } \\
\text { SFA }\end{array}$ & $\begin{array}{l}0.13 \\
0.07\end{array}$ & $\begin{array}{l}0.14 \\
0.17\end{array}$ & $\begin{array}{l}0.18 \\
0.08\end{array}$ & $\begin{array}{l}0.21 \\
0.11\end{array}$ & 0.06 & $\begin{array}{c}-0.15 \\
0.00\end{array}$ & $\begin{array}{c}-0.18 \\
0.03\end{array}$ & $\begin{array}{c}-0.05 \\
0.07\end{array}$ & $\begin{array}{c}-0.10 \\
0.00\end{array}$ & $\begin{array}{l}-0.25 \\
-0.07\end{array}$ & $0.77 \%$ & 0.58 & $0.77 \%$ & $0.76^{*}$ & 0.50 \\
\hline $\begin{array}{l}\text { MAA } \\
\text { MUFA }\end{array}$ & 0.09 & $\begin{array}{l}0.17 \\
0.12\end{array}$ & $\begin{array}{l}0.08 \\
0.18\end{array}$ & $\begin{array}{l}0.11 \\
0.21\end{array}$ & $\begin{array}{l}.05 \\
0.01\end{array}$ & $\begin{array}{l}0.00 \\
-0.16\end{array}$ & $\begin{array}{l}0.03 \\
-0.16\end{array}$ & $\begin{array}{l}0.07 \\
-0.04\end{array}$ & $\begin{array}{c}0.00 \\
-0.04\end{array}$ & $\begin{array}{l}-0.07 \\
-0.24\end{array}$ & $0.82 * *$ & $\begin{array}{l}0.13 \\
0.63\end{array}$ & $\begin{array}{l}0.29 * \\
0.88^{* *}\end{array}$ & $\begin{array}{l}0.45 \\
0.72 *\end{array}$ & 0.28 \\
\hline PUFA & 0.17 & 0.01 & 0.25 & 0.25 & 0.05 & -0.28 & -0.43 & -0.17 & -0.29 & -0.47 & 0.80 ** & $0.77 *$ & $0.88 *$ & $0.74 *$ & 0.49 \\
\hline $\mathrm{n}-3$ fatty acids & 0.33 & 0.11 & 0.29 & 0.22 & 0.25 & 0.13 & -0.09 & 0.08 & 0.03 & 0.02 & 0.66 & 0.80 ** & 0.66 & 0.54 & 0.51 \\
\hline $\mathrm{n}-6$ fatty acids & 0.09 & -0.03 & 0.20 & 0.23 & -0.03 & -0.41 & -0.50 & -0.25 & -0.37 & -0.60 & $0.79^{*}$ & $0.71^{*}$ & $0.88^{* *}$ & $0.75^{*}$ & 0.45 \\
\hline Carbohydrate & -0.15 & -0.01 & -0.10 & -0.05 & -0.08 & 0.05 & 0.13 & 0.12 & 0.31 & 0.14 & -0.62 & -0.54 & -0.56 & $-0.71^{*}$ & -0.48 \\
\hline Sodium & 0.07 & -0.02 & -0.03 & 0.09 & 0.16 & 0.07 & -0.10 & -0.10 & 0.06 & 0.15 & 0.01 & 0.34 & 0.00 & 0.20 & 0.19 \\
\hline $\begin{array}{l}\text { Potassium } \\
\text { Calcium }\end{array}$ & $\begin{array}{l}0.21 \\
0.26\end{array}$ & $\begin{array}{l}0.13 \\
0.16\end{array}$ & $\begin{array}{l}0.03 \\
0.09\end{array}$ & $\begin{array}{l}0.09 \\
0.17\end{array}$ & $\begin{array}{l}0.28 \\
0.37\end{array}$ & $\begin{array}{l}0.15 \\
0.24\end{array}$ & $\begin{array}{l}0.08 \\
0.17\end{array}$ & $\begin{array}{l}-0.04 \\
0.05\end{array}$ & $\begin{array}{l}0.05 \\
0.11\end{array}$ & $\begin{array}{l}0.29 \\
0.36\end{array}$ & 0.30 & $\begin{array}{l}0.53 \\
070\end{array}$ & 0.20 & 0.26 & 0.24 \\
\hline $\begin{array}{l}\text { Calcium } \\
\text { Magnesium }\end{array}$ & $\begin{array}{l}0.26 \\
0.10\end{array}$ & $\begin{array}{l}0.16 \\
-0.01\end{array}$ & $\begin{array}{c}0.09 \\
-0.06\end{array}$ & $\begin{array}{l}0.17 \\
0.05\end{array}$ & $\begin{array}{l}0.37 \\
0.20\end{array}$ & $\begin{array}{l}0.24 \\
0.06\end{array}$ & $\begin{array}{c}0.17 \\
-0.02\end{array}$ & $\begin{array}{l}0.05 \\
-0.13\end{array}$ & $\begin{array}{c}0.11 \\
-0.06\end{array}$ & $\begin{array}{l}0.36 \\
0.18\end{array}$ & $\begin{array}{l}0.21 \\
0.17\end{array}$ & $\begin{array}{l}0.20 \\
0.52\end{array}$ & $\begin{array}{l}0.13 \\
0.09\end{array}$ & $\begin{array}{l}0.50 \\
0.38\end{array}$ & $\begin{array}{l}0.34 \\
0.29\end{array}$ \\
\hline $\begin{array}{l}\text { Magnesium } \\
\text { Phosphorus }\end{array}$ & $\begin{array}{l}0.10 \\
0.05\end{array}$ & $\begin{array}{l}-0.01 \\
0.02\end{array}$ & $\begin{array}{l}-0.00 \\
-0.16\end{array}$ & $\begin{array}{l}0.05 \\
0.03\end{array}$ & $\begin{array}{l}0.20 \\
0.21\end{array}$ & $\begin{array}{l}0.06 \\
0.06\end{array}$ & $\begin{array}{l}-0.02 \\
-0.06\end{array}$ & $\begin{array}{l}-0.15 \\
-0.18\end{array}$ & $\begin{array}{l}-0.00 \\
-0.06\end{array}$ & $\begin{array}{l}0.18 \\
0.20\end{array}$ & $\begin{array}{l}0.17 \\
-0.09\end{array}$ & $\begin{array}{l}0.52 \\
0.24\end{array}$ & $\begin{array}{l}.0 .09 \\
-0.19\end{array}$ & $\begin{array}{l}0.38 \\
0.25\end{array}$ & $\begin{array}{l}0.29 \\
0.23\end{array}$ \\
\hline $\begin{array}{l}17 \text { Iron } \\
\text { Iron }\end{array}$ & $\begin{array}{l}0.001 \\
-0.01\end{array}$ & $\begin{array}{l}0.02 \\
-0.12\end{array}$ & $\begin{array}{l}-0.10 \\
-0.23\end{array}$ & $\begin{array}{l}-0.06 \\
-0.06\end{array}$ & 0.14 & -0.14 & $\begin{array}{l}\quad-0.00 \\
-0.25\end{array}$ & $\begin{array}{l}\quad-1.16 \\
-0.35\end{array}$ & $\begin{array}{l}-0.000 \\
-0.26\end{array}$ & $\begin{array}{l}0.02 \\
-0.02\end{array}$ & -0.04 & $\begin{array}{l}0.24 \\
0.37\end{array}$ & -0.16 & $\begin{array}{l}0.25 \\
0.10\end{array}$ & 0.19 \\
\hline & -0.16 & -0.13 & -0.30 & 0.06 & 0.04 & -0.16 & -0.23 & -0.41 & -0.16 & 0.06 & -0.17 & 0.26 & -0.13 & 0.23 & 0.11 \\
\hline $\mathrm{Co}_{1}$ & -0.04 & -0.13 & -0 . & -0.03 & 0.07 & -0.11 & -0.22 & -0.30 & -0.24 & & -0.04 & & & & 11 \\
\hline Mang & -0.36 & -0.34 & -0.4 & -0.35 & -0.18 & -0.14 & -0.3 & -0 . & -0.16 & -0.24 & -0.52 & -0.08 & -0.64 & -0.44 & 0.09 \\
\hline$\beta$-carotenc & 0.15 & 0.03 & -0.0 & 0.09 & 0.25 & -0.04 & -0.09 & -0.2 & & & 0.1 & 0.41 & 0.2 & 0.01 & -0.04 \\
\hline Vitamin D & & & 0.1 & 0.1 & & $0.2>>$ & & $0.0 \varepsilon$ & & & -0.20 & & & 0.09 & 0.11 \\
\hline$\alpha$-tocopherol eq & 0.21 & 0.03 & 0.10 & 0.17 & 0.24 & -0.25 & -0.31 & -0.30 & -0.11 & -0.12 & $0.68^{*}$ & $0.71 *$ & $0.72 *$ & 0.47 & 0.36 \\
\hline Vitami & 0.02 & -0.10 & -0 . & 0.0 & & -0.26 & -0.34 & -0.42 & -0.38 & -0.16 & 0.07 & 0.36 & 0.10 & 0.25 & 0.07 \\
\hline Vitamir & 0.02 & 0.02 & -0 & -0.02 & & -0.03 & -0.04 & -0.26 & & 0. & 0. & & & 0.14 & 19 \\
\hline Vitamin & -0.01 & -0.05 & -0 . & -0 & 0. & -0.13 & -0.28 & -0.31 & & -0 & & & & 0.40 & 39 \\
\hline Niaci & 0.11 & 0.03 & -0.05 & 0.00 & 0.1 & 0.1 & -0.06 & -0.09 & & & & (1) $>$ & 0.00 & 0.15 & 0.27 \\
\hline $\begin{array}{l}\text { Vitamin B6 } \\
\text { Vitan }\end{array}$ & 0.11 & 0.05 & -0.08 & -0.07 & 0.18 & 0.07 & -0.02 & -0.13 & -0.07 & 0.19 & 0.07 & 34 & -0.06 & -0.07 & 09 \\
\hline $\begin{array}{l}\text { Vitamin B12 } \\
\text { Folicasid }\end{array}$ & 0.18 & 0.04 & 0.01 & 0.03 & 0.24 & 0.15 & -0.06 & -0.04 & -0.03 & 0.14 & -0.14 & 0.28 & -0.14 & 0.12 & 18 \\
\hline $\begin{array}{c}\text { Folic acid } \\
\text { Pantothenic acid }\end{array}$ & $\begin{array}{c}0.11 \\
-0.16\end{array}$ & $\begin{array}{l}0.01 \\
-0.13\end{array}$ & $\begin{array}{l}-0.09 \\
-0.30\end{array}$ & $\begin{array}{c}-0.01 \\
0.06\end{array}$ & $\begin{array}{l}0.25 \\
0.04\end{array}$ & $\begin{array}{l}0.04 \\
-0.16\end{array}$ & $\begin{array}{l}-0.04 \\
-0.23\end{array}$ & $\begin{array}{l}-0.14 \\
-0.41\end{array}$ & $\begin{array}{c}0.04 \\
-0.16\end{array}$ & $\begin{array}{l}0.22 \\
0.06\end{array}$ & $\begin{array}{l}-0.09 \\
-017\end{array}$ & 0.25 & $\begin{array}{l}-0.18 \\
-0.13 \\
\end{array}$ & -0.15 & 0.04 \\
\hline Vitamin C & 0.27 & $\begin{array}{l}-0.15 \\
0.20\end{array}$ & $\begin{array}{l}-0.000 \\
0.09\end{array}$ & $\begin{array}{l}0.06 \\
0.12\end{array}$ & 0. & $\begin{array}{l}-0.16 \\
0.24\end{array}$ & $\begin{array}{l}-0.23 \\
0.18\end{array}$ & $\begin{array}{l}-0.41 \\
0.06\end{array}$ & $\begin{array}{l}-0.16 \\
0.17\end{array}$ & $\begin{array}{l}0.06 \\
0.40\end{array}$ & 0.19 & $\begin{array}{l}0.26 \\
0.44\end{array}$ & $\begin{array}{l}-0.13 \\
0.13\end{array}$ & 0.12 & 0.25 \\
\hline & 0.13 & 0.34 & 0.04 & -0.01 & 0.2 & 0.34 & 0.56 & 0.4 & 0 & 0.4 & -0.33 & -0.45 & -0.59 & -0.63 & -0.11 \\
\hline Alcohol & 0.05 & -0.11 & 0.03 & -0.17 & -0.03 & 0.04 & 0.06 & 0.00 & -0.26 & -0.03 & -0.09 & -0.38 & -0.36 & -0.51 & -0.24 \\
\hline & -0.10 & & -0.15 & & -0.08 & -0.27 & & -0.34 & -0.54 & -0.37 & 0.15 & & & & \\
\hline
\end{tabular}

$\mathrm{TB}$, Total body; cf-PWV, carotid-femoral pulse wave velocity; ba-PWV, brachial-ankle pulse wave velocity; SBP, systolic blood pressure; DBP, diastolic blood pressure; MVPA, moderate-to-vigorous physical activity; SFA, saturated fatty acid; MUFA, monounsaturated fatty acid; PUFA, polyunsaturated fatty acids; Eq, equivalents. ${ }^{\text {a }}$ : All $n=20$, Low $n=9$, High $n=11 .{ }^{*} p<0.05, * * p<0.01$ 


\section{Discussion}

This study was performed to investigate the associations of physical activity and nutrient intake with BMD in middle-aged women with high levels of arterial stiffness. To our knowledge, there have been no previous studies directly comparing physical activity and nutrient intake in middle-aged women with low and high levels of arterial stiffness.

The main results of our study were as follows: 1) in the High-PWV group, total-body, arm and leg BMD were negatively associated with age; 2) in the High-PWV group, a positive partial correlation was found between the number of steps and leg BMD after adjusting for age and BMI; 3 ) in the High-PWV group, partial correlations were observed between MUFA, PUFA, $\alpha$-tocopherol Eq intake and BMD.

In the High-PWV group, BMD was negatively associated with age. Previous studies have demonstrated that early postmenopausal women can be "fast bone losers" (bone loss $>3 \%$ annually) or "slow bone losers" (bone loss $\leq 3 \%$ annually) due to differences in basal bone turnover [45,46]. Sumino et al. reported that PWV was significantly correlated with BMD, but not with age or years since menopause, based on the results of multivariate regression analysis [47]. In addition, inflammatory cytokines derived from atherosclerosis can activate osteoclasts and promote bone resorption [21,48], and endothelial dysfunction due to arteriosclerosis decreases nitric oxide production, which promotes bone formation [16-20]. These results suggest that middle-aged women with high levels of arterial stiffness may have a fast rate of bone loss at menopause because the effects of arteriosclerosis and cytokines were added to the decrease in BMD with aging.

Some studies have highlighted a significant association between the severity of atherosclerosis and BMD [47,49], with the association being specific to the site of the lesion [50]. Among postmenopausal women, daily administration of nitroglycerine ointment (NO donor) has been shown to affect arterial dilation and blood flow, thus increasing bone formation and decreasing bone resorption [51]. These findings suggest that among women with high levels of arterial stiffness, one of the causes of bone loss is reduced blood flow, and it is predicted that BMD can be maintained by improving blood flow.

Evans et al. reported that a 9-month walking program with protein supplementation had no effect on BMD in postmenopausal women [52]. Similarly, Cavanaugh and Cann showed that a 52-week walking exercise program did not prevent the loss of BMD in early postmenopausal women [53]. In general, walking activity provides only a slight increase in load on the bone compared to resistance training, and therefore this type of exercise is less effective in promoting bone formation [54,55]. On the other hand, the positive effects of walking exercise have also been tested in more specific populations. Yamazaki et al. reported that 12 months of moderate walking exercise in postmenopausal women with osteopenia or osteoporosis had a positive effect on maintaining BMD and reducing markers of bone resorption [56]. Kitagawa et al. reported that the number of walking steps showed significant positive correlations with bone parameters and a significant decrease in bone resorption markers in elderly women [57]. These studies suggested that the mechanism underlying the positive effect of walking exercise on BMD appears to involve the suppression of bone resorption. The observations of this study suggested that walking exercise may maintain or slow the loss of BMD in middle-aged women with high levels of arterial stiffness due to improved blood flow and reduced bone resorption.

In the High-PWV group, partial correlations were observed between unsaturated fatty acids (MUFA, PUFA) and $\alpha$-tocopherol eq intake and BMD. These observations can be explained by antioxidant and anti-inflammatory effects.

Saturated fatty acids, levels of which were significantly elevated in the PWV-High group, have been suggested to be atherogenic through induction of endothelial dysfunction [58,59]. Numerous reports have indicated increased oxidative stress and chronic inflammation in patients with atherosclerosis [60-63]. Inflammatory cytokines (TNF, IL-6, IL-1), which contribute to inflammatory responses, have been reported to promote osteoclast differentiation [64-67], and oxidative stress was shown to inhibit osteogenic bone formation and differentiation maturation [21]. The PWV-High group may have elevated levels of oxidative stress and inflammatory cytokines that resulted in increased bone resorption and decreased bone formation. 
Oleic acid, a typical monounsaturated fatty acid, and linoleic acid, an $n-6$ polyunsaturated fatty acid, have been shown to produce anti-inflammatory cytokines (IL-4, IL-10, IL-13) [33,68]. Moreover, nitroalkenes generated from oleic acid and linoleic acid under conditions of oxidative stress can exert anti-inflammatory effects [69].

Zhang et al. investigated whether antioxidant intake was associated with risk of fracture and whether this association was modified by the smoking status that induces excessive oxidative stress. The results indicated that vitamin E (antioxidant) intake was associated with reduced risk of fracture in ever smokers but not in never smokers [70]. Similarly, Melhus et al. examined whether the dietary intake of antioxidant vitamins may modify the increased fracture risk associated with smoking. The odds ratio for fracture among recent smokers with a low intake of vitamin $\mathrm{E}$ was 3.0. In contrast, the OR decreased to 1.1 with a high intake of vitamin E. On the other hand, the influence of vitamin E was less pronounced in former smokers [71].

In addition, Takeshima et al. reported that osteoblast differentiation is inhibited by oxidative stress and rescued by antioxidant treatment with vitamin E [72]. These studies suggest a role of oxidant stress in the adverse effects on the BMD, and that insufficient dietary vitamin E intake substantially increases the risk of fracture in people with high levels of oxidative stress, whereas a more adequate intake seems to have a protective effect. The above results suggest that UFA and vitamin E may suppress osteoclast differentiation and activity in the PWV-High group, but not the PWV-Low group, because the role of UFA and vitamin E intake in BMD may be modified by oxidative stress.

The major limitations of this study were the small sample size, although the nutritional intake and physical activity of this study may reflect mean values for present Japanese middle-aged women [42,73-77]. Future studies should investigate the associate physical activity and nutritional intake to the bone mineral density of women with differences in arterial stiffness with a larger sample size to evaluate the validity. Second, nutrient intake was self-reported by the participants. Although BDHQ is the common dietary assessment method used in Japanese studies, it contains a limited list of food items and is difficult to provide accurate reports of food consumption for the general population. Therefore, nutrient intake was adjusted for total energy intake with the residual method. Furthermore, oxidative stress, antioxidant and inflammatory cytokines, as well as nutrients in the blood have not been investigated. It is unclear whether concentration in blood or dietary intake of these materials is more important in middle-aged women. Moreover, nutrient interactions may also affect the results. Further well-designed studies are needed to understand the mechanism and evaluate the effects of nutrient intake on osteoporosis. Finally, a critical cf-PWV cut-off value of $1000 \mathrm{~cm} / \mathrm{s}$ has been adopted for hypertension management or for prediction of the occurrence of cardiovascular events in elderly people [78-80]. However, the threshold at which arterial stiffness alters osteoporosis has not been established. Further studies are required to estimate the cut-off value at which arterial stiffness impacts osteoporosis in postmenopausal women.

\section{Conclusions}

An understanding of vascular-related bone loss may be necessary for effective and efficient prevention and treatment of osteopenia in middle-aged women. The recommendation of physical activity and nutritional intake interventions for the prevention of osteopenia should include consideration of arterial stiffness.

Author Contributions: K.H. was involved in the experimental design, collected data, performed statistical analyses and wrote the original manuscript draft. T.K. was involved in the conception of the study, collected data, performed statistical analyses and substantively revised the manuscript. M.F. was involved in the experimental design, interpretation of data and substantively revised the manuscript. K.S. (Koji Sato) was involved in data collection. M.I. was involved in data collection and interpretation. T.H. was involved in the design of the study and in data collection and interpretation. K.S. (Kiyoshi Sanada) was involved in the experimental design, data collection and interpretation, substantively revised the manuscript and sourced the research funding. All authors have read and agreed to the published version of the manuscript. 
Funding: This work was supported by a Grant-in-Aid for Scientific Research from the Ministry of Education, Culture, Sports, Science and Technology of Japan (\#24300239, K. Sanada) (https://kaken.nii.ac.jp/d/p/24300239.ja. $\mathrm{html}$ ). The funders had no role in study design, data collection and analysis, decision to publish or preparation of the manuscript. All of the authors have read, reviewed, edited and approved the final manuscript.

Acknowledgments: The authors would like to thank all subjects who participated in this study.

Conflicts of Interest: The authors declare no conflict of interest.

\section{References}

1. Lello, S.; Capozzi, A.; Scambia, G. Osteoporosis and cardiovascular disease: An update. Gynecol. Endocrinol. 2015, 31, 590-594. [CrossRef] [PubMed]

2. Yoshimura, N.; Muraki, S.; Oka, H.; Kawaguchi, H.; Nakamura, K.; Akune, T. Cohort profile: Research on Osteoarthritis/Osteoporosis Against Disability study. Int. J. Epidemiol. 2010, 39, 988-995. [CrossRef] [PubMed]

3. Yoshimura, N.; Muraki, S.; Oka, H.; Mabuchi, A.; En-Yo, Y.; Yoshida, M.; Saika, A.; Yoshida, H.; Suzuki, T.; Yamamoto, S.; et al. Prevalence of knee osteoarthritis, lumbar spondylosis, and osteoporosis in Japanese men and women: The research on osteoarthritis/osteoporosis against disability study. J. Bone Miner Metab. 2009, 27, 620-628. [CrossRef] [PubMed]

4. Kahwati, L.C.; Weber, R.P.; Pan, H.; Gourlay, M.; LeBlanc, E.; Coker-Schwimmer, M.; Viswanathan, M. Vitamin D, Calcium, or Combined Supplementation for the Primary Prevention of Fractures in Community-Dwelling Adults: Evidence Report and Systematic Review for the US Preventive Services Task Force. JAMA J. Am. Med. Assoc. 2018, 319, 1600-1612. [CrossRef]

5. Weaver, C.M.; Gordon, C.M.; Janz, K.F.; Kalkwarf, H.J.; Lappe, J.M.; Lewis, R.; O’Karma, M.; Wallace, T.C.; Zemel, B.S. The National Osteoporosis Foundation's position statement on peak bone mass development and lifestyle factors: A systematic review and implementation recommendations. Osteoporos. Int. J. Establ. Result Coop. Eur. Found. Osteoporos. Natl. Osteoporos. Found. USA 2016, 27, 1281-1386. [CrossRef]

6. Bailey, R.L.; Looker, A.C.; Lu, Z.; Fan, R.; Eicher-Miller, H.A.; Fakhouri, T.H.; Gahche, J.J.; Weaver, C.M.; Mills, J.L. B-vitamin status and bone mineral density and risk of lumbar osteoporosis in older females in the United States. Am J. Clin. Nutr. 2015, 102, 687-694. [CrossRef]

7. Kemmler, W.; von Stengel, S. Dose-response effect of exercise frequency on bone mineral density in post-menopausal, osteopenic women. Scand. J. Med. Sci. Sports 2014, 24, 526-534. [CrossRef]

8. Kemmler, W.; von Stengel, S. Exercise frequency, health risk factors, and diseases of the elderly. Arch. Phys. Med. Rehabil. 2013, 94, 2046-2053. [CrossRef]

9. Wolff, I.; van Croonenborg, J.J.; Kemper, H.C.; Kostense, P.J.; Twisk, J.W. The effect of exercise training programs on bone mass: A meta-analysis of published controlled trials in pre- and postmenopausal women. Osteoporos. Int. J. Establ. Result Coop. Eur. Found. Osteoporos. Natl. Osteoporos. Found. USA 1999, 9, 1-12. [CrossRef]

10. London, G.M.; Marchais, S.J.; Guerin, A.P.; Boutouyrie, P.; Metivier, F.; de Vernejoul, M.C. Association of bone activity, calcium load, aortic stiffness, and calcifications in ESRD. J. Am. Soc. Nephrol. 2008, 19, 1827-1835. [CrossRef]

11. Pelletier, S.; Confavreux, C.B.; Haesebaert, J.; Guebre-Egziabher, F.; Bacchetta, J.; Carlier, M.C.; Chardon, L.; Laville, M.; Chapurlat, R.; London, G.M.; et al. Serum sclerostin: The missing link in the bone-vessel cross-talk in hemodialysis patients? Osteoporos. Int. J. Establ. Result Coop. Eur. Found. Osteoporos. Natl. Osteoporos. Found. USA 2015, 26, 2165-2174. [CrossRef] [PubMed]

12. Giallauria, F.; Ling, S.M.; Schreiber, C.; Maggio, M.; Shetty, V.; Muller, D.; Vigorito, C.; Ferrucci, L.; Najjar, S.S. Arterial stiffness and bone demineralization: The Baltimore longitudinal study of aging. Am. J. Hypertens. 2011, 24, 970-975. [CrossRef] [PubMed]

13. Gold, E.S.; Ramsey, S.A.; Sartain, M.J.; Selinummi, J.; Podolsky, I.; Rodriguez, D.J.; Moritz, R.L.; Aderem, A. ATF3 protects against atherosclerosis by suppressing 25-hydroxycholesterol-induced lipid body formation. J. Exp. Med. 2012, 209, 807-817. [CrossRef] [PubMed]

14. Chou, M.Y.; Fogelstrand, L.; Hartvigsen, K.; Hansen, L.F.; Woelkers, D.; Shaw, P.X.; Choi, J.; Perkmann, T.; Backhed, F.; Miller, Y.I.; et al. Oxidation-specific epitopes are dominant targets of innate natural antibodies in mice and humans. J. Clin. Invest. 2009, 119, 1335-1349. [CrossRef] 
15. Yang, X.; Li, Y.; Li, Y.; Ren, X.; Zhang, X.; Hu, D.; Gao, Y.; Xing, Y.; Shang, H. Oxidative Stress-Mediated Atherosclerosis: Mechanisms and Therapies. Front. Physiol. 2017, 8, 600. [CrossRef]

16. Collin-Osdoby, P.; Nickols, G.A.; Osdoby, P. Bone cell function, regulation, and communication: A role for nitric oxide. J. Cell. Biochem. 1995, 57, 399-408. [CrossRef]

17. Brandi, M.L.; Hukkanen, M.; Umeda, T.; Moradi-Bidhendi, N.; Bianchi, S.; Gross, S.S.; Polak, J.M.; MacIntyre, I. Bidirectional regulation of osteoclast function by nitric oxide synthase isoforms. Proc. Natl. Acad. Sci. USA 1995, 92, 2954-2958. [CrossRef]

18. Lowik, C.W.; Nibbering, P.H.; van de Ruit, M.; Papapoulos, S.E. Inducible production of nitric oxide in osteoblast-like cells and in fetal mouse bone explants is associated with suppression of osteoclastic bone resorption. J. Clin. Invest. 1994, 93, 1465-1472. [CrossRef]

19. Kasten, T.P.; Collin-Osdoby, P.; Patel, N.; Osdoby, P.; Krukowski, M.; Misko, T.P.; Settle, S.L.; Currie, M.G.; Nickols, G.A. Potentiation of osteoclast bone-resorption activity by inhibition of nitric oxide synthase. Proc. Natl. Acad. Sci. USA 1994, 91, 3569-3573. [CrossRef]

20. MacIntyre, I.; Zaidi, M.; Alam, A.S.; Datta, H.K.; Moonga, B.S.; Lidbury, P.S.; Hecker, M.; Vane, J.R. Osteoclastic inhibition: An action of nitric oxide not mediated by cyclic GMP. Proc. Natl. Acad. Sci. USA 1991, 88, 2936-2940. [CrossRef]

21. Parhami, F.; Jackson, S.M.; Tintut, Y.; Le, V.; Balucan, J.P.; Territo, M.; Demer, L.L. Atherogenic diet and minimally oxidized low density lipoprotein inhibit osteogenic and promote adipogenic differentiation of marrow stromal cells. J. Bone Miner. Res. Off. J. Am. Soc.Bone Miner. Res. 1999, 14, 2067-2078. [CrossRef] [PubMed]

22. Parhami, F.; Morrow, A.D.; Balucan, J.; Leitinger, N.; Watson, A.D.; Tintut, Y.; Berliner, J.A.; Demer, L.L. Lipid oxidation products have opposite effects on calcifying vascular cell and bone cell differentiation. A possible explanation for the paradox of arterial calcification in osteoporotic patients. Arterioscler. Thromb. Vasc. Biol. 1997, 17, 680-687. [CrossRef] [PubMed]

23. Tanaka, H.; DeSouza, C.A.; Seals, D.R. Absence of age-related increase in central arterial stiffness in physically active women. Arterioscler. Thromb. Vasc. Biol. 1998, 18, 127-132. [CrossRef] [PubMed]

24. Tanaka, H.; Palta, P.; Folsom, A.R.; Meyer, M.L.; Matsushita, K.; Evenson, K.R.; Aguilar, D.; Heiss, G. Habitual physical activity and central artery stiffening in older adults: The Atherosclerosis Risk in Communities study. J. Hypertens. 2018, 36, 1889-1894. [CrossRef] [PubMed]

25. Boyle, L.J.; Credeur, D.P.; Jenkins, N.T.; Padilla, J.; Leidy, H.J.; Thyfault, J.P.; Fadel, P.J. Impact of reduced daily physical activity on conduit artery flow-mediated dilation and circulating endothelial microparticles. J. Appl. Physiol. (1985) 2013, 115, 1519-1525. [CrossRef]

26. Gando, Y.; Yamamoto, K.; Murakami, H.; Ohmori, Y.; Kawakami, R.; Sanada, K.; Higuchi, M.; Tabata, I.; Miyachi, M. Longer Time Spent in Light Physical Activity Is Associated With Reduced Arterial Stiffness in Older Adults. Hypertension 2010, 56, 540-546. [CrossRef]

27. Steele, B. Physical activity, aging and cardiovascular disease. Can. J. Cardiol. 1999, 15, 849-853.

28. Avenell, A.; Mak, J.C.; O'Connell, D. Vitamin D and vitamin D analogues for preventing fractures in post-menopausal women and older men. Cochrane Database Syst. Rev. 2014. [CrossRef]

29. Kruger, M.C.; Horrobin, D.F. Calcium metabolism, osteoporosis and essential fatty acids: a review. Prog Lipid Res. 1997, 36, 131-151. [CrossRef]

30. Hu, F.B.; Willett, W.C. Optimal diets for prevention of coronary heart disease. JAMA J. Am. Med. Assoc. 2002, 288, 2569-2578. [CrossRef]

31. Vafeiadou, K.; Weech, M.; Altowaijri, H.; Todd, S.; Yaqoob, P.; Jackson, K.G.; Lovegrove, J.A. Replacement of saturated with unsaturated fats had no impact on vascular function but beneficial effects on lipid biomarkers, E-selectin, and blood pressure: Results from the randomized, controlled Dietary Intervention and VAScular function (DIVAS) study. Am. J. Clin. Nutr. 2015, 102, 40-48. [CrossRef] [PubMed]

32. Vaccaro, J.A.; Huffman, F.G. Monounsaturated fatty acid, carbohydrate intake, and diabetes status are associated with arterial pulse pressure. Nutr. J. 2011, 10. [CrossRef] [PubMed]

33. Hall, W.L. Dietary saturated and unsaturated fats as determinants of blood pressure and vascular function. Nutr. Res. Rev. 2009, 22, 18-38. [CrossRef] [PubMed]

34. Iso, H.; Kobayashi, M.; Ishihara, J.; Sasaki, S.; Okada, K.; Kita, Y.; Kokubo, Y.; Tsugane, S.; Group, J.S. Intake of fish and $\mathrm{n} 3$ fatty acids and risk of coronary heart disease among Japanese: The Japan Public Health Center-Based (JPHC) Study Cohort I. Circulation 2006, 113, 195-202. [CrossRef] 
35. Kanis, J.A.; McCloskey, E.V.; Johansson, H.; Oden, A.; Melton, L.J., III; Khaltaev, N. A reference standard for the description of osteoporosis. Bone 2008, 42, 467-475. [CrossRef]

36. Looker, A.C.; Melton, L.J., III; Harris, T.; Borrud, L.; Shepherd, J.; McGowan, J. Age, gender, and race/ethnic differences in total body and subregional bone density. Osteoporos. Int. J. Establ. Result Coop. Eur. Found. Osteoporos. Natl. Osteoporos. Found. USA 2009, 20, 1141-1149. [CrossRef]

37. Hasegawa, N.; Fujie, S.; Horii, N.; Uchida, M.; Kurihara, T.; Sanada, K.; Hamaoka, T.; Iemitsu, M. Aerobic exercise training-induced changes in serum C1q/TNF-related protein levels are associated with reduced arterial stiffness in middle-aged and older adults. Am. J. Physiol. Regul. Integr. Comp. Physiol. 2018, 314, R94-R101. [CrossRef]

38. Zempo-Miyaki, A.; Fujie, S.; Sato, K.; Hasegawa, N.; Sanada, K.; Maeda, S.; Hamaoka, T.; Iemitsu, M. Elevated pentraxin 3 level at the early stage of exercise training is associated with reduction of arterial stiffness in middle-aged and older adults. J. Hum. Hypertens. 2016, 30, 521-526. [CrossRef]

39. Yamamoto, K.; Kawano, H.; Gando, Y.; Iemitsu, M.; Murakami, H.; Sanada, K.; Tanimoto, M.; Ohmori, Y.; Higuchi, M.; Tabata, I.; et al. Poor trunk flexibility is associated with arterial stiffening. Am. J. Physiol. Heart Circ. Physiol. 2009, 297, H1314-H1318. [CrossRef]

40. Kurita, S.; Yano, S.; Ishii, K.; Shibata, A.; Sasai, H.; Nakata, Y.; Fukushima, N.; Inoue, S.; Tanaka, S.; Sugiyama, T.; et al. Comparability of activity monitors used in Asian and Western-country studies for assessing free-living sedentary behaviour. PLoS ONE 2017, 12, e0186523. [CrossRef]

41. Tanaka, C.; Fujiwara, Y.; Sakurai, R.; Fukaya, T.; Yasunaga, M.; Tanaka, S. Locomotive and non-locomotive activities evaluated with a triaxial accelerometer in adults and elderly individuals. Aging Clin. Exp. Res. 2013, 25, 637-643. [CrossRef] [PubMed]

42. Kobayashi, S.; Honda, S.; Murakami, K.; Sasaki, S.; Okubo, H.; Hirota, N.; Notsu, A.; Fukui, M.; Date, C. Both comprehensive and brief self-administered diet history questionnaires satisfactorily rank nutrient intakes in Japanese adults. J. Epidemiol. 2012, 22, 151-159. [CrossRef] [PubMed]

43. Sasaki, S.; Yanagibori, R.; Amano, K. Self-administered diet history questionnaire developed for health education: A relative validation of the test-version by comparison with 3-day diet record in women. J. Epidemiol. 1998, 8, 203-215. [CrossRef] [PubMed]

44. Willett, W.C.; Howe, G.R.; Kushi, L.H. Adjustment for total energy intake in epidemiologic studies. Am J. Clin. Nutr. 1997, 65, 1220S-1228S, discussion 1229S-1231S. [CrossRef]

45. Hansen, M.A.; Overgaard, K.; Riis, B.J.; Christiansen, C. Role of peak bone mass and bone loss in postmenopausal osteoporosis: 12 year study. BMJ 1991, 303, 961-964. [CrossRef]

46. Christiansen, C.; Riis, B.J.; Rodbro, P. Prediction of rapid bone loss in postmenopausal women. Lancet 1987, 1, 1105-1108. [CrossRef]

47. Sumino, H.; Ichikawa, S.; Kasama, S.; Takahashi, T.; Kumakura, H.; Takayama, Y.; Kanda, T.; Sakamaki, T.; Kurabayashi, M. Elevated arterial stiffness in postmenopausal women with osteoporosis. Maturitas 2006, 55, 212-218. [CrossRef]

48. Parhami, F.; Tintut, Y.; Beamer, W.G.; Gharavi, N.; Goodman, W.; Demer, L.L. Atherogenic high-fat diet reduces bone mineralization in mice. J. Bone Miner. Res. Off. J. Am. Soc.Bone Miner. Res. 2001, 16, 182-188. [CrossRef]

49. Baykara, M.; Ozturk, C.; Elbuken, F. The relationship between bone mineral density and arterial stiffness in women. Diagn. Interv. Radiol. 2012, 18, 441-445. [CrossRef]

50. Fehervari, M.; Sarkadi, H.; Krepuska, M.; Sotonyi, P.; Acsady, G.; Entz, L.; Lakatos, P.; Szeberin, Z. Bone Mineral Density is Associated with Site-Specific Atherosclerosis in Patients with Severe Peripheral Artery Disease. Calcif. Tissue Int. 2013, 93, 55-61. [CrossRef]

51. Jamal, S.A.; Hamilton, C.J.; Eastell, R.; Cummings, S.R. Effect of nitroglycerin ointment on bone density and strength in postmenopausal women: A randomized trial. JAMA J. Am. Med. Assoc. 2011, 305, 800-807. [CrossRef] [PubMed]

52. Evans, E.M.; Racette, S.B.; Van Pelt, R.E.; Peterson, L.R.; Villareal, D.T. Effects of soy protein isolate and moderate exercise on bone turnover and bone mineral density in postmenopausal women. Menopause 2007, 14, 481-488. [CrossRef] [PubMed]

53. Cavanaugh, D.J.; Cann, C.E. Brisk walking does not stop bone loss in postmenopausal women. Bone 1988, 9 , 201-204. [CrossRef] 
54. Gomez-Cabello, A.; Ara, I.; Gonzalez-Aguero, A.; Casajus, J.A.; Vicente-Rodriguez, G. Effects of training on bone mass in older adults: A systematic review. Sports Med. 2012, 42, 301-325. [CrossRef] [PubMed]

55. Nikander, R.; Sievanen, H.; Heinonen, A.; Daly, R.M.; Uusi-Rasi, K.; Kannus, P. Targeted exercise against osteoporosis: A systematic review and meta-analysis for optimising bone strength throughout life. BMC Med. 2010, 8, 47. [CrossRef]

56. Yamazaki, S.; Ichimura, S.; Iwamoto, J.; Takeda, T.; Toyama, Y. Effect of walking exercise on bone metabolism in postmenopausal women with osteopenia/osteoporosis. J. Bone Miner Metab. 2004, 22, 500-508. [CrossRef]

57. Kitagawa, J.; Nakahara, Y. Associations of daily walking steps with calcaneal ultrasound parameters and a bone resorption marker in elderly Japanese women. J. Physiol Anthropol. 2008, 27, 295-300. [CrossRef]

58. American Heart Association Nutrition Committee; Lichtenstein, A.H.; Appel, L.J.; Brands, M.; Carnethon, M.; Daniels, S.; Franch, H.A.; Franklin, B.; Kris-Etherton, P.; Harris, W.S.; et al. Diet and lifestyle recommendations revision 2006: A scientific statement from the American Heart Association Nutrition Committee. Circulation 2006, 114, 82-96. [CrossRef]

59. Vogel, R.A.; Corretti, M.C.; Plotnick, G.D. Effect of a single high-fat meal on endothelial function in healthy subjects. Am. J. Cardiol. 1997, 79, 350-354. [CrossRef]

60. Pant, S.; Deshmukh, A.; Gurumurthy, G.S.; Pothineni, N.V.; Watts, T.E.; Romeo, F.; Mehta, J.L. Inflammation and atherosclerosis-revisited. J. Cardiovasc. Pharmacol. Ther. 2014, 19, 170-178. [CrossRef]

61. Kattoor, A.J.; Pothineni, N.V.K.; Palagiri, D.; Mehta, J.L. Oxidative Stress in Atherosclerosis. Curr. Atheroscler. Rep. 2017, 19, 42. [CrossRef] [PubMed]

62. Harrison, D.; Griendling, K.K.; Landmesser, U.; Hornig, B.; Drexler, H. Role of oxidative stress in atherosclerosis. Am. J. Cardiol. 2003, 91, 7A-11A. [CrossRef]

63. Geovanini, G.R.; Libby, P. Atherosclerosis and inflammation: Overview and updates. Clin. Sci. (Lond.) 2018, 132, 1243-1252. [CrossRef] [PubMed]

64. Nguyen, M.T.; Favelyukis, S.; Nguyen, A.K.; Reichart, D.; Scott, P.A.; Jenn, A.; Liu-Bryan, R.; Glass, C.K.; Neels, J.G.; Olefsky, J.M. A subpopulation of macrophages infiltrates hypertrophic adipose tissue and is activated by free fatty acids via Toll-like receptors 2 and 4 and JNK-dependent pathways. J. Biol. Chem. 2007, 282, 35279-35292. [CrossRef] [PubMed]

65. Akatsu, T.; Takahashi, N.; Udagawa, N.; Imamura, K.; Yamaguchi, A.; Sato, K.; Nagata, N.; Suda, T. Role of prostaglandins in interleukin-1-induced bone resorption in mice in vitro. J. Bone Miner. Res. Off. J. Am. Soc. Bone Miner. Res. 1991, 6, 183-189. [CrossRef] [PubMed]

66. Lacey, D.L.; Timms, E.; Tan, H.L.; Kelley, M.J.; Dunstan, C.R.; Burgess, T.; Elliott, R.; Colombero, A.; Elliott, G.; Scully, S.; et al. Osteoprotegerin ligand is a cytokine that regulates osteoclast differentiation and activation. Cell 1998, 93, 165-176. [CrossRef]

67. Udagawa, N.; Takahashi, N.; Katagiri, T.; Tamura, T.; Wada, S.; Findlay, D.M.; Martin, T.J.; Hirota, H.; Taga, T.; Kishimoto, T.; et al. Interleukin (IL)-6 induction of osteoclast differentiation depends on IL-6 receptors expressed on osteoblastic cells but not on osteoclast progenitors. J. Exp. Med. 1995, 182, 1461-1468. [CrossRef]

68. Carmen Naranjo, M.; Garcia, I.; Bermudez, B.; Lopez, S.; Cardelo, M.P.; Abia, R.; Muriana, F.J.G.; Montserrat-de la Paz, S. Acute effects of dietary fatty acids on osteclastogenesis via RANKL/RANK/OPG system. Mol. Nutr. Food Res. 2016, 60, 2505-2513. [CrossRef]

69. Hwang, J.; Lee, K.E.; Lim, J.Y.; Park, S.I. Nitrated fatty acids prevent TNFalpha-stimulated inflammatory and atherogenic responses in endothelial cells. Biochem. Biophys. Res. Commun. 2009, 387, 633-640. [CrossRef]

70. Zhang, J.; Munger, R.G.; West, N.A.; Cutler, D.R.; Wengreen, H.J.; Corcoran, C.D. Antioxidant intake and risk of osteoporotic hip fracture in Utah: An effect modified by smoking status. Am. J. Epidemiol. 2006, 163, 9-17. [CrossRef]

71. Melhus, H.; Michaelsson, K.; Holmberg, L.; Wolk, A.; Ljunghall, S. Smoking, antioxidant vitamins, and the risk of hip fracture. J. Bone Miner. Res. Off. J. Am. Soc.Bone Miner. Res. 1999, 14, 129-135. [CrossRef] [PubMed]

72. Takeshima, K.; Nishiwaki, Y.; Suda, Y.; Niki, Y.; Sato, Y.; Kobayashi, T.; Miyamoto, K.; Uchida, H.; Inokuchi, W.; Tsuji, T.; et al. A missense single nucleotide polymorphism in the ALDH2 gene, rs671, is associated with hip fracture. Sci. Rep. 2017, 7, 428. [CrossRef] [PubMed]

73. Inoue, S.; Ohya, Y.; Tudor-Locke, C.; Yoshiike, N.; Shimomitsu, T. Step-defined physical activity and cardiovascular risk among middle-aged Japanese: The National Health and Nutrition Survey of Japan 2006. J. Phys. Act. Health 2012, 9, 1117-1124. [CrossRef] [PubMed] 
74. Htun, N.C.; Suga, H.; Imai, S.; Shimizu, W.; Ishikawa-Takata, K.; Takimoto, H. Dietary pattern and its association with blood pressure and blood lipid profiles among Japanese adults in the 2012 Japan National Health and Nutrition Survey. Asia Pac. J. Clin. Nutr. 2018, 27, 1048-1061. [CrossRef] [PubMed]

75. Nakamura, M.; Tajima, S.; Yoshiike, N. Nutrient Intake in Japanese Adults From the National Nutrition Survey, 1995-99. J. Nutr. Sci. Vitaminol. 2002, 48, 433-441. [CrossRef]

76. Mikumo, M.; Okano, H.; Yoshikata, R.; Ishitani, K.; Ohta, H. Association between lumber bone mineral density and vascular stiffness as assessed by pulse wave velocity in postmenopausal women. J. Bone Miner. Metab. 2009, 27, 89-94. [CrossRef]

77. Sanada, K.; Iemitsu, M.; Murakami, H.; Gando, Y.; Kawano, H.; Kawakami, R.; Tabata, I.; Miyachi, M. Adverse effects of coexistence of sarcopenia and metabolic syndrome in Japanese women. Eur. J. Clin. Nutr. 2012, 66, 1093-1098. [CrossRef]

78. Williams, B.; Mancia, G.; Spiering, W.; Agabiti Rosei, E.; Azizi, M.; Burnier, M.; Clement, D.; Coca, A.; De Simone, G.; Dominiczak, A.; et al. 2018 Practice Guidelines for the management of arterial hypertension of the European Society of Cardiology and the European Society of Hypertension. Blood Press 2018, 27, 314-340. [CrossRef]

79. Van Bortel, L.M.; Laurent, S.; Boutouyrie, P.; Chowienczyk, P.; Cruickshank, J.K.; De Backer, T.; Filipovsky, J.; Huybrechts, S.; Mattace-Raso, F.U.; Protogerou, A.D.; et al. Expert consensus document on the measurement of aortic stiffness in daily practice using carotid-femoral pulse wave velocity. J. Hypertens. 2012, 30, 445-448. [CrossRef]

80. Butlin, M.; Qasem, A. Large Artery Stiffness Assessment Using SphygmoCor Technology. Pulse 2017, 4, 180-192. [CrossRef]

(C) 2020 by the authors. Licensee MDPI, Basel, Switzerland. This article is an open access article distributed under the terms and conditions of the Creative Commons Attribution (CC BY) license (http://creativecommons.org/licenses/by/4.0/). 